\title{
Embedding Power Line Communication in Photovoltaic Optimizer by Modulating Data in Power Control Loop
}

\author{
Yue Zhu, Jiande Wu, Member, IEEE, Ruichi Wang, Student Member, IEEE, \\ Zhengyu Lin, Senior Member, IEEE, and Xiangning He, Fellow, IEEE
}

\begin{abstract}
In Photovoltaic (PV) system, dc-dc power optimizer (DCPO) is an option to maximize output power. At the same time, data links among DCPOs are often required for system monitoring and controlling. This paper proposes a novel power line communication (PLC) method for the DCPOs, in which the data of a DCPO is modulated into the control loop of power converter, and then transmitted through the series-connected dc power line to other DCPOs. In the process of communication, differential phase shift keying (DPSK) modulation and discrete Fourier transformation (DFT) demodulation are employed. To analyze the quality of communication, the communication model of the system is built, based on small-signal model. Furthermore, the noises of the system, including switching, maximum power point tracking (MPPT) and additive white Gaussian noise (AWGN), are discussed and measured to evaluate the signal-to-noise ratio (SNR). At last, an experimental system including 6 DCPOs is established and tested, which verifies the feasibility and effectiveness of the proposed method.
\end{abstract}

Index Terms-Photovoltaic (PV) system, Power line communication, DC power optimizer.

\section{INTRODUCTION}

$\mathrm{P}$ HOTOVOLTAIC (PV) generation has been one of the most popular renewable energy technologies in the world. Conventionally, dozens of panels are series-connected to increase the output voltage, and then feeds to a grid-tied inverter. The output currents of all panels are equal due to the series connection. However, the current generation of PV panel is dependent on solar intensity and its condition. If some panels generate less current than the string current due to partial shading, the unshaded panels have to lower their output current to match with the shaded ones. Consequently, the unshaded panels will not work at their maximum power point (MPP), which leads to a waste of power [1]. Such problem can also be

Manuscript received February 4, 2018; revised April 24, 2018; accepted May 4, 2018. This work is sponsored by the National Nature Science Foundation of China under Grants 51577170, National Key Technologies R\&D Program of China No. 2017YFE0112400, and the European Union's Horizon 2020 research and innovation programme under grant agreement No. 734796.

Y. Zhu, J. Wu, R. Wang and X. He are with the College of Electrical Engineering, Zhejiang University, Hangzhou; 310027, China. (e-mail: y_zhu@foxmail.com; eewjd@zju.edu.cn; rui_chi@163.com;hxn@zju.edu.cn).

Z. Lin is with the Department of Electrical, Electronic and Power Engineering, Aston University, Birmingham, B4 7ET, U.K. (email: z.lin@ieee.org). induced by manufacture difference, nonuniform aging, etc.

To mitigate the aforementioned problem, dc-dc power optimizer (DCPO), which is a dc-dc converter with input connected to the PV panel and output connected to other DCPOs in series, has been proposed and widely studied [1]-[9]. By equipping each PV panel with a DCPO, the panel can work independently at its MPP, thus distributed maximum power point tracking can be achieved.

In [1]-[4], different topologies of DCPO have been compared and analyzed. Buck, boost, buck-boost, etc., are candidate topologies for DCPO. Buck and boost converters are the most efficient and low-cost topologies [2][3], while buck is only suited for long string and boost is for short strings. Buck-boost, cuk and sepic converters are flexible in voltage ranges but always at an efficiency and alternatively cost shortcoming [1][2][4]. In [5]-[7], modeling of a single DCPO and of a PV string equipped with DCPOs have been studied in detail, which is very helpful for system designing. In [8], a novel maximum power point tracking (MPPT) technique based on DCPO is proposed, which shows the effectiveness of DCPO in panel MPPT. [9] proposes an intelligent PV module concept based on DCPO and PLC, and it shows great potentials of DCPO in intelligent PV system development.

In a PV system equipped with DCPOs, PV status monitoring, intelligent control algorithm, and outage management can be implemented only if communication links among DCPOs are provided. Due to the cost-sensitive characteristic of PV system, low-cost communication method is preferred. For conventional PV status monitoring systems, wireless communication is commonly used [10]-[14], in which ZigBee technique gets more attention. In [14], ZigBee is employed in a distributed PV generator due to the advantages of low power consumption and cost-effectiveness. However, if the Wi-Fi access point is deployed in the same area, it may corrupt ZigBee packet and cause severe interference [19]. Meanwhile, the vulnerable characteristic of wireless communication may damage the security operation of PV system. Thus, wireless communication is not the best scheme for PV system.

DC PLC technology, which utilizes the dc power line as an alternative communication channel, is considered as a viable communication method in distribution power systems. Due to its reliable and low-cost advantages [15], dc PLC has become an attractive solution for PV monitoring [16]-[21]. In [16], a low-cost low-frequency PLC for PV monitoring was developed, using baseband transmission and direct spread code division multiple access (DS-CDMA). However, the achieved bit rate in 
this literature is $5 \mathrm{bps}$, which can only be employed in low-demand PV monitoring occasions. In [20], A capacitive coupling PLC scheme is proposed. A coupling transformer is utilized to transmit signal carrier wave. However, due to the parallel connection, the transmission efficiency is low, which results in high transmitting power. In [21], a parallel resonant coupling unit is designed to increase the transmission efficiency of dc PLC, and 10kbps bit rate and low error rate are achieved. However, since the coupling unit is series-connected with the string and the generating current of PV panels flows through it, the efficiency of the power conversion would decrease slightly. [9] introduces an approach which integrates PLC with DCPO. However, in this scheme, the DCPO and PLC circuits are designed independently, hence the total cost and volume are increased compared with a mere DCPO. In general, conventional PLC design consists of three components, including a signal source, coupling circuits, and a demodulation circuit. To reduce cost, it would be ideal if DCPO and PLC can share a common circuit.

Data communication can be integrated with power converter, and transmitted with the power either sequentially [22]-[23] or simultaneously [24]-[27]. [22] proposed a novel PLC scheme in which power electronic circuits transmit power in discontinues mode, at the period of power off, data signal can be transmitted at the same lines. [23] optimizes this sequential transmitting scheme and achieves a maximum bit rate of $4.68 \mathrm{Mbps}$. However, in sequential transmission strategy, power is transmitted as pulse in the power line. In long distance situation, e.g. PV system, transmission line effect will significantly interfere the signal and lead to data corruption [22]. Therefore, sequential transmission strategy is unsuitable for PV system. In the aspect of simultaneous transmission strategy, [24] proposed a PLC scheme among dc-dc converters sharing a common input dc bus, in which the communication signal is generated by switching frequency modulation of power converters. [25]-[27] investigate the common method that embedding data signal into pulse width modulation (PWM), and term it power and signal dual modulation (PSDM) technique. The systems adopting PSDM technique can reduce cost and simplify circuitry, which corresponds to the requirement of PV system. According to the studies, switching frequency and the pulse phase of the converter are the common elements to carry information. [25] realizes communication using frequency shift keying (FSK) modulation on PWM carrier, while [26] employs phase shift keying (PSK) modulation and direct sequence spread spectrum (DSSS) technique on PWM carrier. The carrier-modulated converter can send out data signal, but the amplitude of the signal is small and cannot be regulated, thus it is restricted in long-range communication applications, including PV system. [27] proposes a PSDM method that can regulate the amplitude of the carrier, but this method is based on phase shift full bridge (PSFB) converter and is hard to apply in other topologies.

This paper proposes a new PSDM method for PV system equipped with DCPOs: modulating data in power control loop of DCPOs by digital controller and demodulating the data from the outputs of other DCPOs.

The contributions of this paper are as follows.

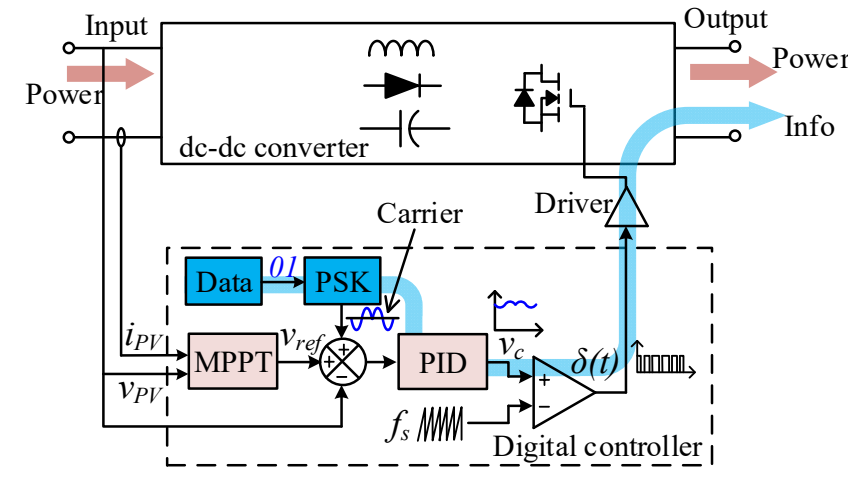

(a)

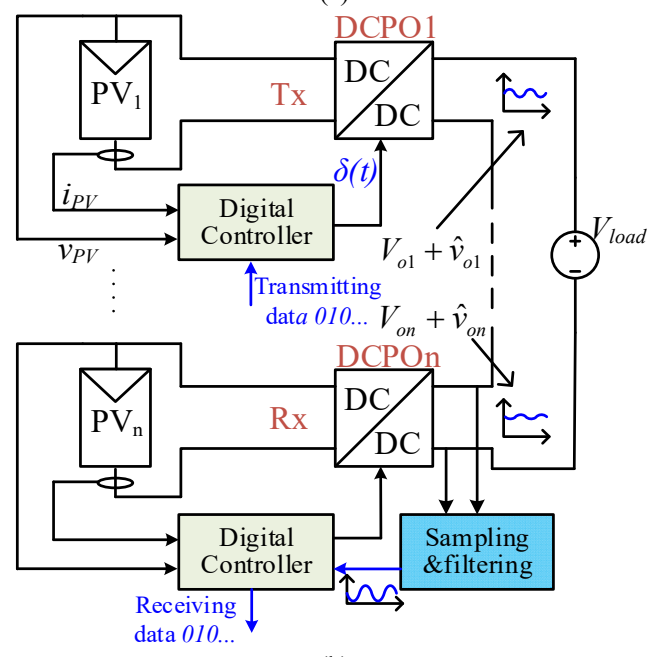

(b)

Fig. 1. (a) Structure of signal modulation in a single DCPO's power control loop. (b) Structure of the proposed communication method in a PV string.

1) A novel PLC method for PV system is presented, in which the data signal is modulated through the power control loop of the DCPO and sent out from the output of DCPO.

2)The model of the communication system is built, and the noise influences from switching process, MPPT and additive white Gaussian noise (AWGN) in the PV system are analyzed. 3) An experimental system is built, and a reliable communication with $2 \mathrm{kbps}$ bit rate has been achieved, which verifies the correctness of this method.

\section{Principle and Design of the Modulating Method}

\section{A. Principle}

For a PV system equipped with DCPOs, a data link among DCPOs is necessary for PV status monitoring, including input and output voltages and currents, temperature, and other parameters. Besides, innovative control algorithm may be developed if data is exchanged among DCPOs.

For industrial application, considering the conversion efficiency and cost issue, buck topology is identified as the most cost-efficient topology for DCPO.

Fig. 1(a) shows the structure of a single DCPO with data modulation. The DCPO is originally utilized to maximize the output power of PV panel. A common DCPO control strategy is employed, in which MPPT is implemented by regulating PV output voltage and measuring PV output power. The controller of the DCPO regulates the PV voltage to follow a time-variant 


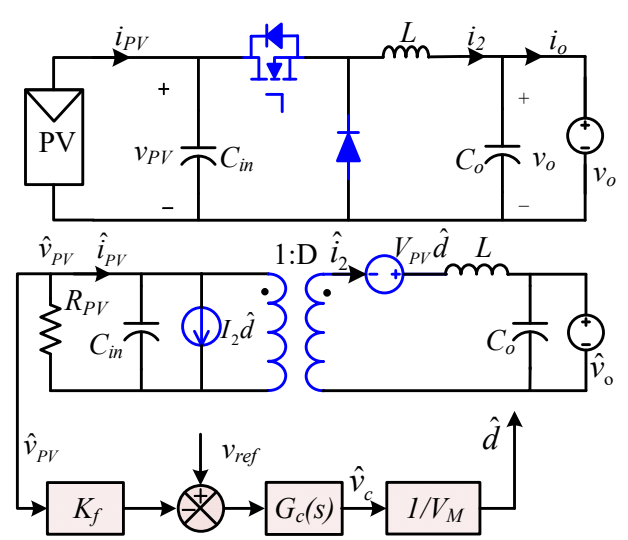

Fig. 2. Topology and small-signal model of a single buck DCPO

value $v_{\text {ref, }}$ which is continuously updated by MPPT algorithm. However, in a view of communication system, it can also be exploited as a data transmitter. If data carrier is added to the reference voltage $v_{\text {ref }}$ of the power control loop, a perturbation carrying data information will be introduced in the duty-cycle $d$. Thus, the data will be modulated at the output voltage of DCPO and transmitted via the cascaded power line. From the view of communication, the DCPO with data modulation is regarded as a data transmitter (Tx) and the power line of the PV string is a communication channel. Meanwhile, the other DCPOs in the string are the receivers (Rxs).

Fig. 1(b) shows the system structure which applies the proposed PLC scheme. A string composed of $n$ panels with associated DCPOs is presented in the system. As a first-order approximation, it is possible to model the PV inverter as a voltage source $V_{\text {load }}$ with a series resistance [5].The resistance is ignored in this paper because the value is small comparing with the impedance of DCPOs in the string.

The following discussion is based on the scenario that DCPO1 is sending out data, and the other DCPOs are receiving. When a sinusoidal wave is added to $v_{\text {ref }}$ of DCPO1, a voltage perturbation $\hat{v}_{o 1}$ with the same frequency will be produced at the output. Meanwhile, the voltage perturbation will transmit to the outputs of other DCPOs and can be extracted by voltage sampling circuits. Assuming that the DCPOs have the same output impedance, the relationship between $\hat{v}_{o 1}$ and output perturbation of receivers $\hat{v}_{o i}$ is

$$
\hat{v}_{o i}=-\hat{v}_{o 1} /(n-1) \quad i \in\{2,3 \ldots, n\}
$$

where symbols with hats represent small-signal variations around the steady state values. $\hat{v}_{o 1}$ is the output voltage perturbation of transmitter, and $\hat{v}_{o i}$ is the voltage perturbation at the output of $i^{\text {th }}$ DCPO.

Three major problems should be analyzed before applying this scheme.

First, to keep the communication reliable under different situations, the signal amplitude at output voltage should be regulated by the digital controller. Therefore, the gain of signal carrier amplitude from digital controller to output voltage must be calculated.
Second, the impact to PV power system should be evaluated. Since the PV module is not a voltage source, the data signal modulated in control loop not only generates a perturbation at the output of the DCPO, but also introduces a disturbance at the input of the DCPO, which may result in performance degradation of the PV panel. Thus, it is significant that the disturbance at the input should be small while the perturbation at the output should be large.

Third, multiple noises, including switching process and MPPT algorithm, influence the communication reliability and increases bit error rate (BER). Therefore, noise analysis is necessary to give a reference for selecting proper signal amplitude.

Further study focuses on these problems and gives analysis in detail.

\section{B. Modeling}

This paper selects buck converter for further study due to its low cost and high efficiency advantages. A series string connection of buck DCPOs allows total independence of output voltage and power. Besides, the internal diode helps the converter get bypassed automatically while the module is inactive [2]. Nevertheless, a minimum number of PV panels is required to guarantee the dc bus voltage while under extreme non-homogeneous irradiance conditions, which is regarded as a constraint of buck DCPO application.

In the following part, a buck converter with input voltage feedback control is employed. MPPT process is not concerned for modeling.

For DCPOs, the circuit parameters and feedback network are designed for power conversion primarily. In a well-designed DCPO, data modulation should be designed according to the following principles.

1) The signal gain from reference to output voltage should be large to acquire large signal amplitude, whilst the gain from signal reference to input voltage should be small to reduce the influence on PV panel.

2) The carrier frequency should be high to achieve a high bit rate. And it would be better if switching frequency is integral multiple of the carrier wave frequency, which will be explained in Section III-B.

Small-signal model of a DCPO is essential for analysis. The schematic of a single DCPO under study and the small-signal model are shown in Fig. 2, where the equivalent series resistance (ESR) of the inductor and capacitor are ignored. $R_{P V}$ is the dynamic resistance of PV module at MPP and can be expressed as

$$
\frac{1}{R_{P V}}=\left.\frac{\partial i_{P V}}{\partial v_{P V}}\right|_{M P P}
$$

To simplify the power control loop design of DCPO, the output voltage is assumed as a constant, thus $\hat{v}_{o}$ is considered as zero. The open-loop transfer functions of a single DCPO from $\hat{d}$ to $\hat{v}_{p v}$ is derived by [7]

$$
G_{v_{P V} d_{-} O L}(s)=\left.\frac{\hat{v}_{P V}(s)}{\hat{d}(s)}\right|_{\hat{v}_{o}=0}=\frac{K_{o}\left(s+a_{o}\right)}{s^{2}+2 \xi_{o} \omega_{o} s+\omega_{o}^{2}}
$$

where 


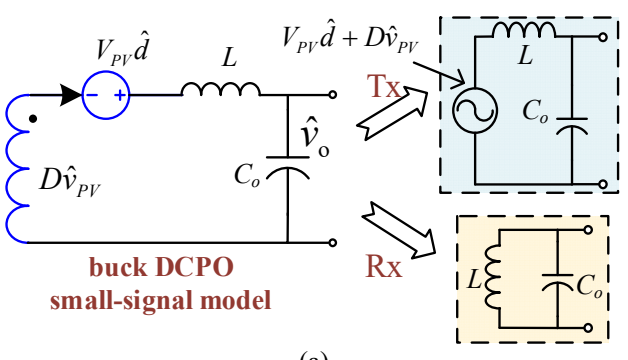

(a)

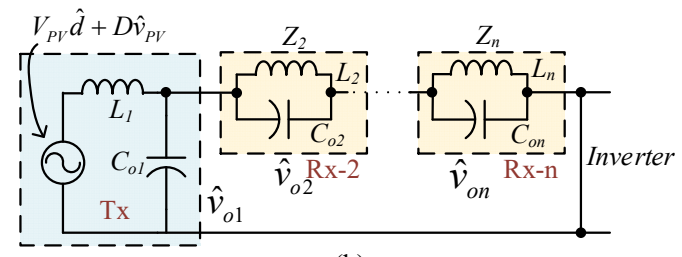

(b)

Fig. 3. (a) The simplified model of Tx and Rx. (b) The model of the communication system.

$$
\left\{\begin{array}{l}
K_{o}=-\frac{I_{o}}{C_{i n}} \\
a_{o}=\frac{V_{P V} D}{L I_{o}} \\
\omega_{o}=\frac{D}{\sqrt{L C_{i n}}} \\
\xi_{o}=-\frac{1}{2 D R_{P V}} \sqrt{\frac{L}{C_{i n}}}
\end{array} .\right.
$$

From the poles and zeros of $G_{v_{P V} d O L}(s)$, a lead-lag compensator function $G_{c}(s)$ is designed as

$$
G_{c}(s)=-\frac{K_{\mathrm{c}}\left(s+\omega_{o} / 2\right)^{2}}{s\left(s+a_{o}\right)\left(s+2 \pi f_{s}\right)}
$$

In a feedback control system, the loop transfer function is

$$
T_{c}(s)=K_{f} G_{c}(s) G_{v_{p v} d_{-} O L}(s) / V_{M}
$$

where $K_{f}$ is the feedback factor, and $V_{M}$ is the amplitude of PWM sawtooth wave. The value of $K_{C}$ decides the gain-crossover frequency of $T_{c}(s)$. Thus, for a single buck DCPO, the close-loop transfer function from $\hat{d}$ to $\hat{v}_{p v}$ is

$$
G_{v_{P V} d_{-} C L}(s)=\frac{G_{c}(s) G_{v p v d_{-} O L}(s) / V_{M}}{1+T_{c}(s)} .
$$

In the communication system, since the DCPO is a data transmitter, and the carrier wave is modulated on the output of the DCPO, $\hat{v}_{o}$ should not be considered as zero as before. Therefore, both $\hat{v}_{p v}$ and $\hat{v}_{o}$ are state variables, which are influenced by input variable $\hat{d}$. The transfer functions from $\hat{d}$ to $\hat{v}_{p v}$ and $\hat{d}$ to $\hat{v}_{o}$ are of high order, but it can be simplified by superposition principle. Assuming $\hat{d}$ is zero, the transfer function from $\hat{v}_{o}$ to $\hat{v}_{p v}$ can be derived from Fig. 2 and written as

$$
\left.G_{v_{P V} v_{o}}(s)\right|_{\hat{d}=0}=\frac{D}{s^{2} L C_{i n}-s \frac{L}{R_{P V}}+D^{2}} .
$$

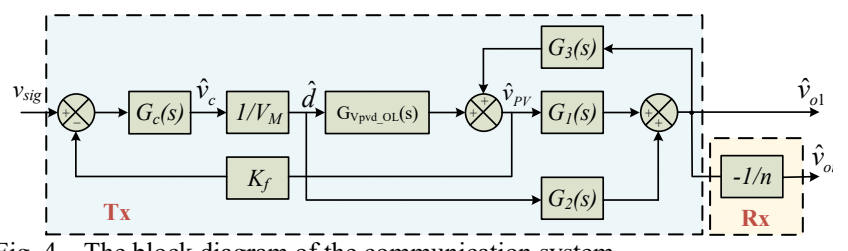

Fig. 4. The block diagram of the communication system.

In such way, from (7) and (8), $\hat{v}_{P V}$ is derived as

$$
\hat{v}_{P V}=G_{v_{v_{V} d_{-} C L}}(s) \hat{d}+\left.G_{v_{P V} v_{o}}(s)\right|_{\hat{d}=0} \hat{v}_{\mathrm{o}}
$$

To calculate $\hat{v}_{o}$ precisely, the close-loop impedance of every DCPO should be deduced, which is complicated, and the detailed derivation is given by [5]. However, in the communication model, signal carrier frequency is much higher than the crossover frequency $f_{g}$ of $T_{c}(s)$. Feedback network of Rx will not response to such signal. Therefore, for $\mathrm{Rx}, \hat{d}$ will be zero, and the dependent voltage source $V_{P V} \hat{d}$ can be regarded as shorted. Meanwhile, if the input capacitor $C_{i n}$ is large, the perturbation at the input $\hat{v}_{P V}$ of $\mathrm{Rx}$ will be small, and $D \hat{v}_{P V}$ is approximately equal to zero. Thus, $\mathrm{Rx}$ can be regarded as a parallel LC branch, which greatly simplifies the calculation, as shown in Fig. 3(a). The impedance of each $\mathrm{Rx}$ is

$$
Z_{2}=Z_{3}=\cdots=Z_{n}=\frac{s L}{s^{2} L C+1} \text {. }
$$

The simplified communication system is shown in Fig. 3(b). Using superposition principle, the Tx output perturbation $\hat{v}_{o 1}$ is derived as (11), and the $\mathrm{Rx}$ output perturbation $\hat{v}_{o i}$ is derived as (12)

$$
\begin{gathered}
\hat{v}_{o 1}=\left(D \hat{v}_{p v}+V_{p v} \hat{d}\right) \frac{s C_{o} / /(n-1) Z_{n}}{s L+s C_{o} / /(n-1) Z_{n}} \\
=\frac{n-1}{n} \frac{V_{p v}}{s^{2} L C_{\mathrm{o}}+1} \hat{d}+\frac{n-1}{n} \frac{D}{s^{2} L C_{\mathrm{o}}+1} \hat{v}_{p v} \\
\hat{v}_{o i}=-\frac{1}{n-1} \hat{v}_{o 1} \quad i \in\{2,3, \cdots n\} .
\end{gathered}
$$

Fig. 4 shows a block diagram of the proposed communication system, in which a signal carrier $v_{\text {sig }}$ is modulated into the power control loop. $G_{1}(s), G_{2}(s)$, and $G_{3}(s)$ are the directly transfer functions from $\hat{v}_{p v}$ to $\hat{v}_{o}$, from $\hat{d}$ to $\hat{v}_{o}$, and from $\hat{v}_{o}$ to $\hat{v}_{p v}$, respectively. From (8) and (11), $G_{1}(s), G_{2}(s)$, and $G_{3}(s)$ are calculated as

$$
\begin{gathered}
G_{1}(s)=\frac{n-1}{n} \frac{D}{s^{2} L C_{o}+1} \\
G_{2}(s)=\frac{n-1}{n} \frac{V_{P V}}{s^{2} L C_{o}+1} \\
G_{3}(s)=\frac{D}{s^{2} L C_{i n}-s \frac{L}{R_{P V}}+D^{2}} .
\end{gathered}
$$

For the case study, the PV module parameters and the buck converter parameters are listed in TABLE I. 
TABLE I

PARAMETERS OF THE SYSTEM

\begin{tabular}{lll}
\hline \hline \multirow{2}{*}{ Symbol } & \multicolumn{1}{c}{ Parameter } & \multicolumn{1}{c}{ Value } \\
& \multicolumn{1}{c}{ PARAMETERS OF THE SYSTEM } \\
\hline $\mathrm{S}$ & Solar intensity & $1000 \mathrm{~W} / \mathrm{m}^{2}$ \\
$V_{M P P}$ & Maximum power point voltage & $21.8 \mathrm{~V}$ \\
$I_{M P P}$ & Maximum power point current & $3.56 \mathrm{~A}$ \\
$R_{P V}$ & Dynamic resistance at MPP & $6.70 \Omega$ \\
$f_{s}$ & Converter switching frequency & $50 \mathrm{kHz}$ \\
$n$ & Number of PV panels in the string & 6 \\
$L$ & Converter inductance & $35 \mu \mathrm{H}$ \\
$C_{i n}$ & Input capacitance & $200 \mu \mathrm{F}$ \\
$C_{o}$ & Output capacitance & $22 \mu \mathrm{F}$ \\
$D$ & Steady duty cycle & 0.80 \\
$V_{o}$ & Output voltage & $17.4 \mathrm{~V}$ \\
$I_{o}$ & Output current & $4.45 \mathrm{~A}$ \\
$K_{f}$ & Feedback factor & 1 \\
$K_{C}$ & Coefficient of the compensator network & $1.32 \times 10^{5}$ \\
$V_{M}$ & Amplitude of PWM sawtooth wave & $1 \mathrm{~V}$ \\
$f_{g}$ & Gain-crossover frequency of $T c(s)$ & $2.53 \times 10^{3} \mathrm{~Hz}$ \\
\hline \hline
\end{tabular}

With the help of PSIM ac sweep, bode diagrams of $\hat{v}_{P V} / \hat{v}_{\text {sig }}$ and $\hat{v}_{o} / \hat{v}_{\text {sig }}$ are shown in Fig. 5(a). Since phase shifting from input to output does not affect signal transmitting, only amplitude response is depicted. Switching model bode diagrams are also depicted, which show that the simplified communication model is corresponding with switching model around carrier frequency. In the diagram, the proper range of carrier frequency is from $2 \mathrm{kHz}$ to $10 \mathrm{kHz}$, in which the amplitude gain, from $\hat{v}_{P V}$ to $\hat{v}_{o}$, is about $20 \mathrm{~dB}$. It means that when the signal is modulated into the power control loop of the DCPO, a large perturbation will be produced at the output, while the input perturbation will be small. Consequently, the influence on PV panel will be slight. If carrier frequency is above $10 \mathrm{kHz}$, communication may still work, but small-signal model is imprecise because perturbation frequency is more than $1 / 5$ switching frequency.

The gain from $\hat{v}_{P V}$ to $\hat{v}_{o}$ at different frequency is shown in Fig. 5(b) based on the simplified model. According to above analysis, $10 \mathrm{kHz}$ is selected as carrier wave frequency in this paper. The ratio from $\hat{v}_{o}$ to $\hat{v}_{P V}$ when modulating the signal in the reference voltage is

$$
20 \lg \left(\frac{\hat{v}_{o}}{\hat{v}_{P V}}\right)=6.1-(-13.2)=19.3 \mathrm{~dB} \text {. }
$$

Assuming in a string with $6 \mathrm{DCPOs}, 0.5 \mathrm{~V}_{\text {amp }}$ sinusoidal wave $v_{\text {sig }}$ is modulated into the power control loop of Tx. Then, $1.03 \mathrm{~V}$ amplitude sinusoidal wave will be generated at the output voltage of Tx. At the same time, the amplitude of input voltage perturbation will be $0.11 \mathrm{~V}$, which only produce a slight influence on PV panel.

Different solar intensities and mismatch situations will not influence signal receiving, since the $\mathrm{Rx}$ is taken as a parallel LC branch. For Tx, using the same system model and linear analysis tool, $20 \lg \left(\hat{v}_{o} / \hat{v}_{P V}\right)$ under different solar intensities are depicted in Fig. 5(c), which shows that the gain is even larger in shading condition.

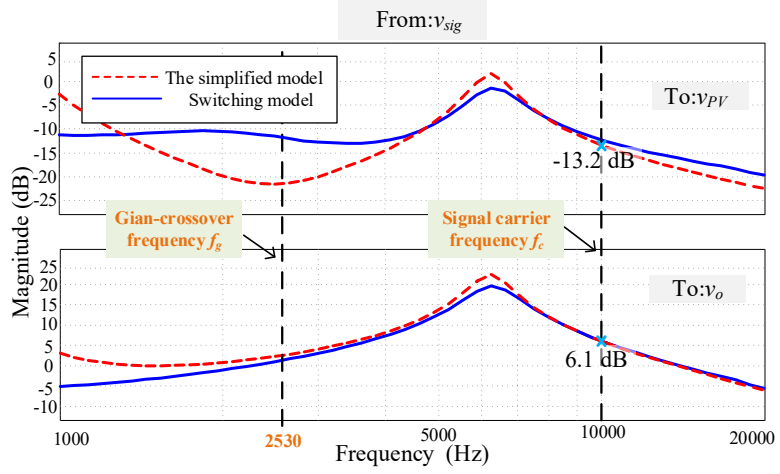

(a)

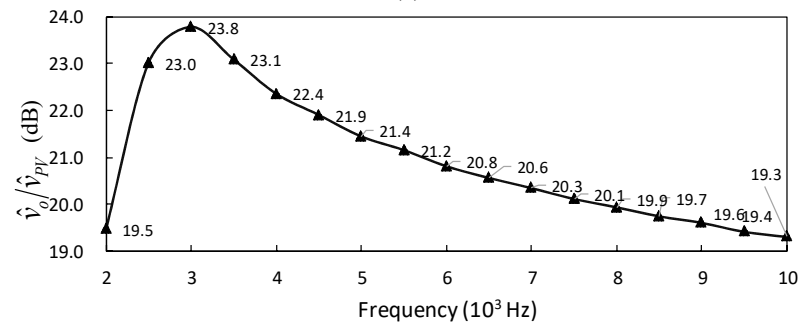

(b)

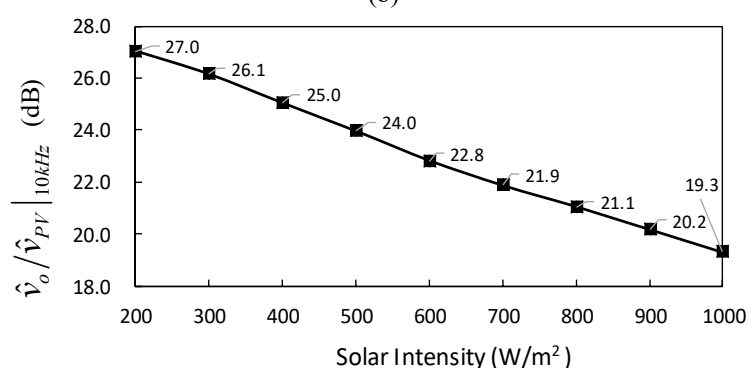

(c)

Fig. 5. (a) Bode diagram of transfer functions from $v_{s i g}$ to $v_{P V}$ and from $v_{s i g}$ to $v_{o}$. (b) Gain under different carrier frequency. (c) Gain at $10 \mathrm{kHz}$ under different solar intensity.

\section{Power Loss Analysis}

The embedded communication signal would introduce additional power loss for the DCPO, and the transmitting signal would consume power, thus, it is necessary to analyze the power loss of the system.

To calculate the power loss, the voltage-perturbation functions of $v_{P V}$ and $v_{o}$ are defined as

$$
\begin{gathered}
\hat{v}_{P V}=A_{P V} \cos \left(\omega_{c} t+\theta_{1}\right) \\
\hat{v}_{o 1}=A_{o} \cos \left(\omega_{c} t+\theta_{2}\right) .
\end{gathered}
$$

For the transmitter, due to the swing of PV working point caused by communication carrier, the additional loss is calculated by [6]

$$
\hat{P}_{i}=\frac{\hat{v}_{P V}^{2}}{R_{M P P}} .
$$

Thus, the power loss for the DCPO can be calculated as

$$
\Delta P_{i}=\frac{1}{T_{c}} \int_{0}^{T_{c}} \frac{A_{P V}^{2} \cos ^{2}\left(\omega_{c} t+\theta_{1}\right)}{R_{P V}} d t=\frac{A_{P V}^{2}}{2 R_{P V}} .
$$

For the communication system, comparing with the impedance of DCPOs, the resistance of DC power line $R_{l}$ is much small, thereby the output current of the carrier is deduced as 


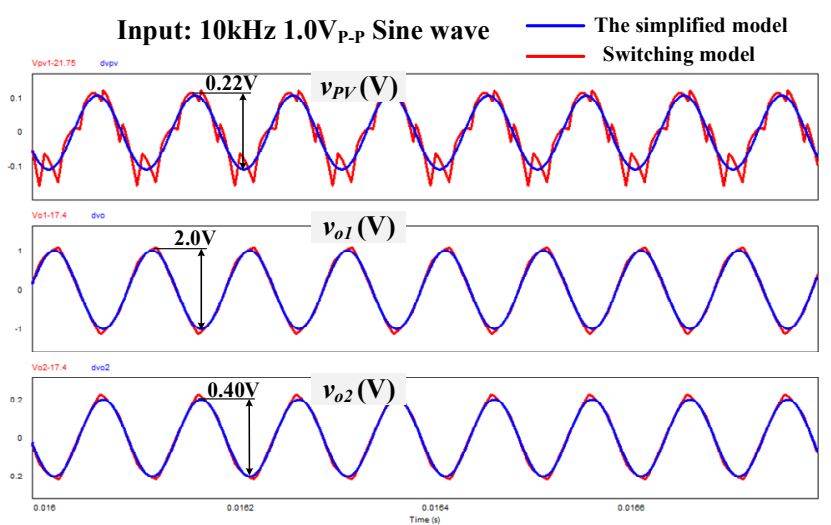

Fig. 6. Simulation result of $v_{P V}, v_{o}$ of DCPO1(Tx) and $v_{o}$ of DCPO2(Rx) in small-signal model and switching model.

$$
\hat{i}_{o}=\frac{\hat{v}_{o 1}}{(n-1) \cdot\left(s L / / \frac{1}{s C_{o}}\right)}=\frac{4 \pi^{2} f_{c}^{2} L C_{o}-1}{(n-1) \cdot 2 \pi f_{c} L} \cdot A_{o} \sin \left(\omega_{c} t+\theta_{2}\right) .
$$

For expression simplification, let

$$
A_{I o}=A_{o} \frac{4 \pi^{2} f_{c}^{2} L C_{o}-1}{(n-1) \cdot 2 \pi f_{c} L} .
$$

Thus, the transmitting power consumption on the power line is

$$
\Delta P_{o}=\frac{1}{T_{c}} \int_{0}^{T_{c}} \hat{i}_{o}^{2} R_{l} d t=\frac{A_{I o}^{2} R_{l}}{2}
$$

and the total power loss for data transmission is

$$
\Delta P_{\text {total }}=\Delta P_{i}+\Delta P_{o} \text {. }
$$

\section{Modeling Verification}

System simulation is implemented to check the proposed small-signal model. The simulation software used is PSIM 9.0, and the parameters are as shown in TABLE I. Six DCPOs are connected in series, and a perturbation is added to the reference voltage of DCPO1. The simulation result is shown in Fig. 6 . The red line shows the output of switching model, which is the result using switching circuit. The blue line shows the output of small-signal model. It proves the accuracy of the model.

\section{PASSBAND TRANSMISSION AND NOISE ANALYSIS}

\section{A. Data Modulation and Demodulation}

To modulate baseband data in the PV string, three basic modulation methods can be exploited, including ASK, FSK and PSK. This paper employs binary differential phase shift keying (2DPSK) method due to the features of narrow bandwidth, high anti-noise performance and easy implementation, as shown in Fig. 7(a).

Non-coherent demodulation and coherent demodulation are generally used in DPSK communication. However, coherent demodulation requires a phase-synchronized clock signal between the transmitter and receivers, which is complex for implementation. Therefore, this paper employs non-coherent orthogonal demodulation method. Still, bit-synchronization is required, which can be easily achieved by program [28].

For $\mathrm{Rx}$, the received signal $s(t)$ after band pass filter (BPF) is

$$
s(t)=A \cos \left(\omega_{c} t+\theta\right) \quad \theta \in[0,2 \pi]
$$

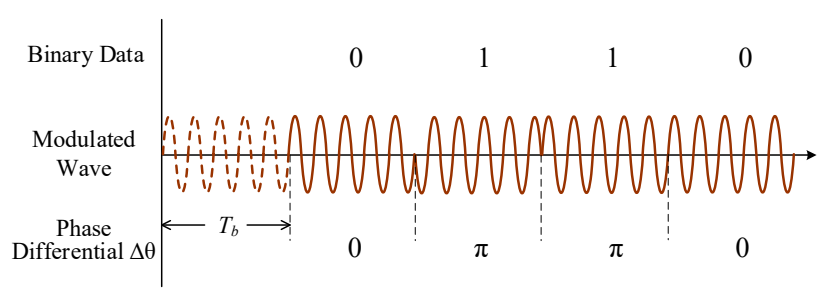

(a)

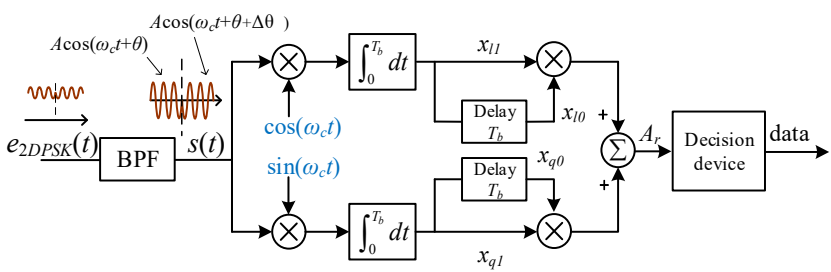

(b)

Fig. 7. (a) Illustrative waveform for 2DPSK. (b) Block diagram of 2DPSK receiver.

where $A, \omega_{c}, \theta$ are the amplitude, angular frequency and relative phase of the carrier wave after BPF, respectively. A symbol is modulated into 5 cycles of carrier wave. For $10 \mathrm{kHz}$ carrier wave, the symbol rate $R_{B}$ will be $2 \mathrm{kBaud}$. As binary, the bit rate of this communication system could reach $2 \mathrm{kbps}$. The period of one-bit $T_{b}$ is

$$
T_{b}=5 T_{c}
$$

where $T_{c}$ is the period of carrier wave. The bandwidth of the carrier B is

$$
B=\frac{2}{T_{b}} .
$$

The procedure diagram of modulation is depicted in Fig. 7(b). Separating the signal by two orthometric vectors $\cos \left(\omega_{c} t\right)$ and $\sin \left(\omega_{c} t\right)$, the reflection values $x_{l}$ and $x_{q}$ are calculated as

$$
\begin{gathered}
x_{l}=\int_{0}^{T_{b}} A \cos \left(\omega_{c} t+\theta\right) \cos \left(\omega_{c} t\right) d t=\left(A T_{b} \cos \theta\right) / 2 \\
x_{q}=\int_{0}^{T_{b}} A \cos \left(\omega_{c} t+\theta\right) \sin \left(\omega_{c} t\right) d t=-\left(A T_{b} \sin \theta\right) / 2 .
\end{gathered}
$$

Considering sequence $s_{0}(t)$ and $s_{1}(t)$, which represents a bit in a continuous data stream, and has a differential phase $\Delta \theta$, the reflection values are $\left(x_{l 0}, x_{q 0}\right),\left(x_{l 1}, x_{q 1}\right)$ respectively. Using (30), the result $A_{r}$ is calculated, and then tested by the decision device according to (31). For bit 0 , the phase stays the same, which means $\Delta \theta=0$, thus, $A_{r}>0$. Conversely, for bit $1, A_{r}<0$. Therefore, decisions of bit data can be made by the value of $A_{r}$.

$$
\begin{gathered}
A_{r}=x_{l 0} x_{l 1}+x_{q 0} x_{q 1}=\left(A^{2} T_{b}^{2} \cos \Delta \theta\right) / 4 \\
\text { data }=\left\{\begin{array}{ll}
0 & \left(A_{r}>0\right) \\
1 & \left(A_{r}<0\right)
\end{array} .\right.
\end{gathered}
$$

The demodulation processes, including A/D conversion, discrete Fourier transform(DFT) calculation and bit decision, are all implemented in MCU by software. For the hardware of demodulator, only a voltage sampling circuit and a BPF circuit are needed, hence the cost of the receiver is very low.

\section{$B$. Noise Analysis}

Noise analysis is vital for a communication system. In the proposed system, many types of interferences are involved, including switching noise, MPPT interference, and white noise. Grid influence, which introduces a $100 \mathrm{~Hz}$ ripple on the dc bus, 


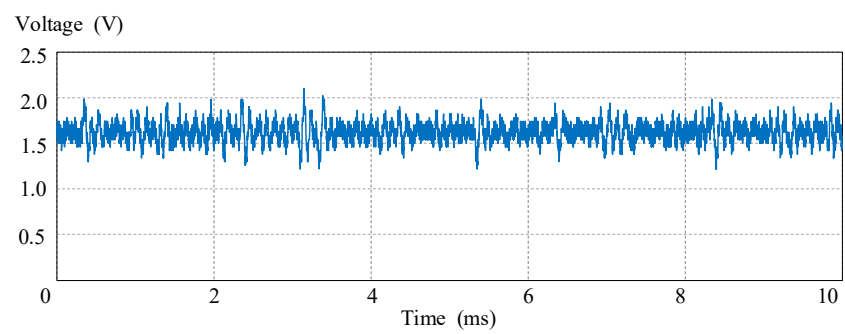

(a)

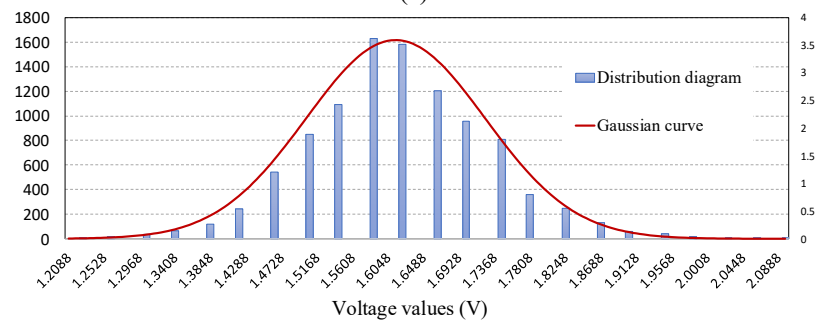

(b)

Fig. 8. (a) Noise sampling at receiving device. (b) The distribution diagram and density function curve of samples.

is not concerned in this paper because it is far from the center frequency of BPF.

For the receiver, the switching noise is mainly from the converter itself, including fundamental wave, harmonics and their sidebands, and the voltage spikes caused by power switching. Most of them are filtered by the BPF, especially the high frequency harmonics. Sidebands of switching frequency will also be filtered due to the high frequency. Voltage spikes can be avoided by separating sampling with switching actions in a PWM period. Thus, switching fundamental wave $g_{s}(t)$ is the major part, which can be written as (32), where $\varphi_{s}$ and $f_{s}$ are the relative angle and frequency of the converter respectively. When $f_{s}$ is integral multiple of the carrier frequency $f_{c}$, the demodulation results will be zero, as shown in (33) and (34). Therefore, the influence of switching fundamental can be eliminated.

$$
\begin{gathered}
g_{s}(t)=A_{s} \cos \left(2 \pi f_{s} t+\varphi_{s}\right) \\
x_{l s}=\int_{0}^{T_{b}} g_{s}(t) \cos \left(\omega_{c} t\right) d t=0 \\
x_{q s}=\int_{0}^{T_{b}} g_{s}(t) \sin \left(\omega_{c} t\right) d t=0 .
\end{gathered}
$$

Perturbation and observation (P\&O) method is the most popular MPPT method in applications. Consequently, a low frequency perturbation around the MPP is introduced. In this paper, to simulate the noise environment, each DCPO produces a $0.1 \mathrm{~V}$ perturbation at $v_{P V}$ every millisecond. At the output of the DCPO, a mixture of MPPT perturbation from itself and other DCPOs, together with white noise, makes the noise randomly and complicated. However, after sampling the noise voltage wave at BPF, as shown in Fig. 8(a), a distribution diagram of the values shows that the mixed noise $n(t)$ is Gaussian, as shown in Fig. 8(b), and the density function is

$$
f_{Y}(y)=\frac{1}{\sqrt{2 \pi} \sigma} \exp \left[-\frac{(y-\mu)^{2}}{2 \sigma^{2}}\right]
$$

where $\mathrm{Y}$ is the random variable, $y$ is the possible value of $Y$, $\mu$ is the mean and $\sigma^{2}$ is variance of the samples, and $\sigma^{2}$ can be regarded as noise power. In this situation, the receiving envi-
TABLE II

PERFORMANCE PARAMETERS OF COMMUNICATION

\begin{tabular}{lll}
\hline \hline Symbol & \multicolumn{1}{c}{ Parameter } & \multicolumn{1}{c}{ Value } \\
\hline$T_{c}$ & Signal carrier wave period & $10 \mu \mathrm{s}$ \\
$T_{b}$ & One-bit period & $50 \mu \mathrm{s}$ \\
$R_{B}$ & Symbol rate & $2 \mathrm{kBaud}$ \\
$\mathrm{B}$ & Bandwidth & $4 \mathrm{kHz}$ \\
$A$ & Signal Amplitude after BPF & $1.0 \mathrm{~V}$ \\
$\sigma^{2}$ & Noise power & $0.0123 \mathrm{~W}$ \\
SNR & Signal-to-Noise Ratio & $16.1 \mathrm{~dB}$ \\
\hline \hline
\end{tabular}

ronment is the same as a sine wave plus narrowband noise. As a result, the signal-to-noise ratio (SNR) can be written as

$$
\frac{S}{N}=10 \lg \left(\frac{A^{2}}{2 \sigma^{2}}\right)
$$

The quantities related to the communication system are listed in TABLE II. The SNR is $16.1 \mathrm{~dB}$, which is a large value. Therefore, the operations of DCPOs in the string will not seriously affect communication effectiveness.

In applications, the imperfection of BPF, the inaccuracy of $\mathrm{ADC}$, and the deviation of bit-synchronization may cause demodulation error. However, these influences are slight, thus they are not discussed in this paper.

\section{EXPERIMENTAL VERIFICATION}

An experimental system, which consists of six DCPOs in series and a constant voltage (CV) load at the terminal, is designed to verify the proposed method, the circuit connection of the system is shown in Fig. 9(a). In the DCPO, buck converter is employed, and controlled by a STM32F407 MCU operating in $168 \mathrm{MHz}$. The picture of the system and the DCPO board are shown in Fig. 9(b) and (c) respectively. To build a stable experimental platform, the PV module is substituted by a $46.6 \mathrm{~V}$ voltage source and a $6.7 \Omega$ resistance connected in series. Other parameters of the system have been shown in TABLE I. By programming, a sinusoidal wave, with $0.5 \mathrm{~V}$ amplitude and $10 \mathrm{kHz}$ frequency, is added to the reference voltage of power control loop of DCPO1. A dc electronic load (CHROMA 6314) is employed as the CV load, which keeps the bus voltage at $104.4 \mathrm{~V}$. The total power of the system is $466 \mathrm{~W}$. To imitate the MPPT process of a real PV system, a $500 \mathrm{~Hz} / 0.1 \mathrm{~V}_{\text {amp }}$ square wave is added to the reference voltage of each DCPO except Tx.

For Tx, data signal is directly modulated to the buck converter by software in the MCU, and no hardware circuit is added. For Rx, a differential amplifier and a BPF are used to extract the signal from the output voltage of the buck converter, and the demodulation process is achieved by MCU program, as shown in Fig. 10.

When the data carrier is modulated into the power control loop of $\mathrm{Tx}(\mathrm{DCPO} 1)$, a perturbation is generated upon the dc voltage. Setting that $\mathrm{Tx}$ is sending out a continuous carrier, the detailed waveforms of perturbation $\hat{v}_{P V}$ and $\hat{v}_{o}$ of Tx, together with $\hat{v}_{P V}$ and $\hat{v}_{o}$ of $\mathrm{Rx}$, are shown in Fig. 11. To measure the perturbations, these waveforms are recorded by ac-coupling of oscilloscope. The accurate amplitude of $10 \mathrm{kHz}$ sinusoidal wave is acquired using FFT function of the oscilloscope. The am- 


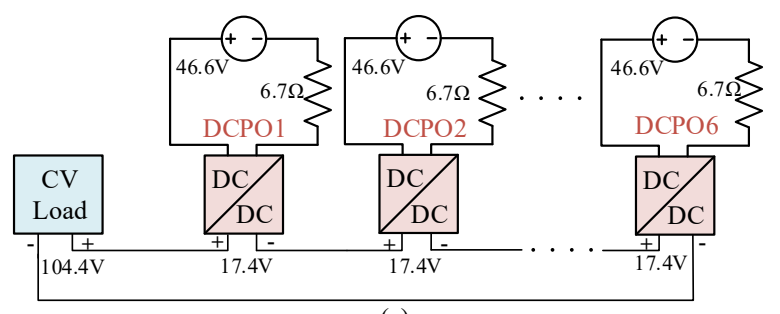

(a)

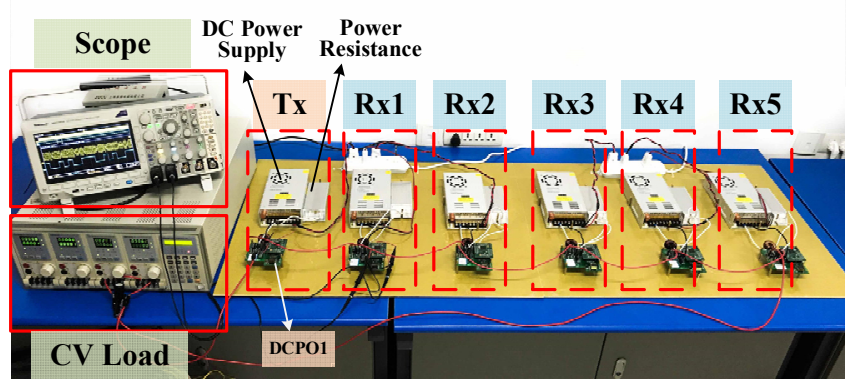

(b)

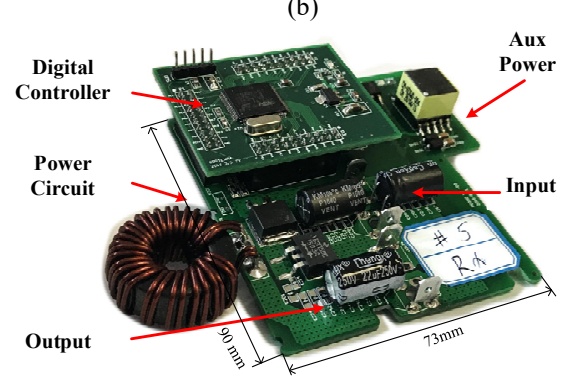

(c)

Fig. 9. (a) Circuit connection of the system. (b) Picture of experimental platform. (c) Picture of the DCPO.

plitude of $\hat{v}_{o}$ is $1.21 \mathrm{~V}$, while the amplitude of $\hat{v}_{P V}$ is $0.13 \mathrm{~V}$. The result agrees with the analysis. The ratio $20 \lg \left(\hat{v}_{P V} / \hat{v}_{o}\right)$ is $19.4 \mathrm{~dB}$ The error between experimental result and theoretical result is $0.1 \mathrm{~dB}$, which proves the accuracy of modeling. The amplitude of voltage perturbation at output of $\mathrm{Rx}$ is $0.20 \mathrm{~V}$. The value is less than $\hat{v}_{o-T x} / 5$, mainly because part of the signal voltage is dropped on the CV load. The amplitude of voltage perturbation at input of $\mathrm{Rx}$ is $0.01 \mathrm{~V}$, which is too small to affect the operations of $\mathrm{Rx}$, thus it can be ignored.

Fig. 12 shows the output voltage of $\mathrm{Tx}$ and $\mathrm{Rx}$, and the demodulation at $\mathrm{Rx}$ when $\mathrm{Tx}$ is sending a data frame with binary sequence 10110010 at the beginning. The amplitude of carrier wave after BPF reaches $1.0 \mathrm{~V}$, and correct sequence is demodulated at Rx using DFT program. The first five cycles of carrier wave, named as pilot-carrier, is used to provide a reference phase for the coming sequence. Moreover, the pilot-carrier is used by program for bit-synchronization.

BER is an important feature to the quality of communication. To estimate BER, Tx is set to transmit $2 \times 10^{5}$ bits in a form of m-sequence, in which symbol 0 and symbol 1 show in equal probability and look randomly. During the test, only one-bit error occurs, which shows the BER is lower than $10^{-5}$.

Experiments under different situations have been implemented to verify the reliability. For buck-DCPO, shoot-through is a common state, in which the duty-cycle of buck converter is 1 and the switching is always on. To simulate this situation, all

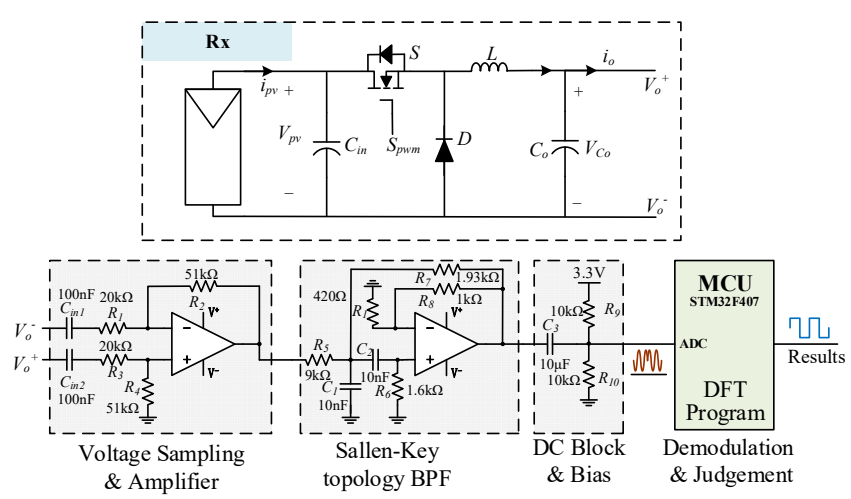

Fig. 10. Demodulation process of Rx.

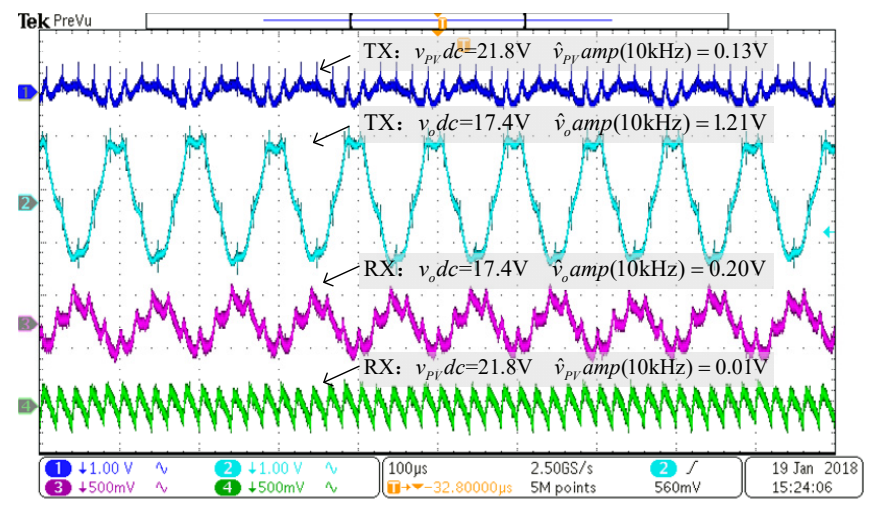

Fig. 11. Waveform of $\hat{v}_{P V}, \hat{v}_{o}$ of Tx, and $\hat{v}_{P V}, \hat{v}_{o}$ of Rx.

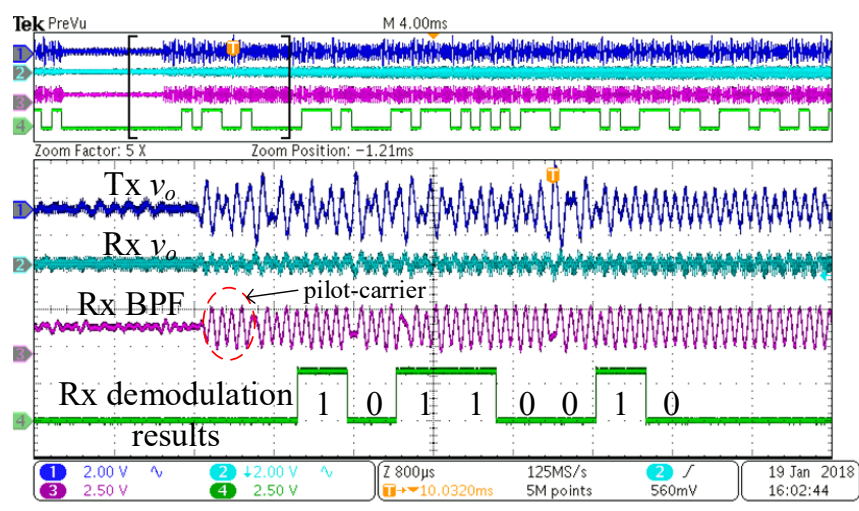

Fig. 12. Waveform of $\hat{v}_{o}$ of Tx and Rx, waveform at BPF, and data demodulation results at a standard state.

the five Rxs are set to work in shoot-though state. The dc bus voltage is changed to $131 \mathrm{~V}$. For Tx, the output voltage couldn't be larger than $21.8 \mathrm{~V}$, hence the dc reference voltage of $\mathrm{Tx}$ changes into $21.3 \mathrm{~V}$ when transmitting signal. Experimental results of $\hat{v}_{o}$ at $\mathrm{Tx}$ and $\mathrm{Rx}$, and demodulation results at $\mathrm{Rx}$ are shown in Fig. 13(a). All the data are demodulated correctly. Because Rxs are working at shoot-through, no switching ripple appears at the output of Rx except those from Tx. Thus, the noise environment is even better at shoot-through state.

In partial shading situation, the MPP of the PV panel will be changed. In most cases, the current will decrease severely. For the DCPO, to match its output current with the string current, the converter will decrease its duty-cycle. Meanwhile, other 


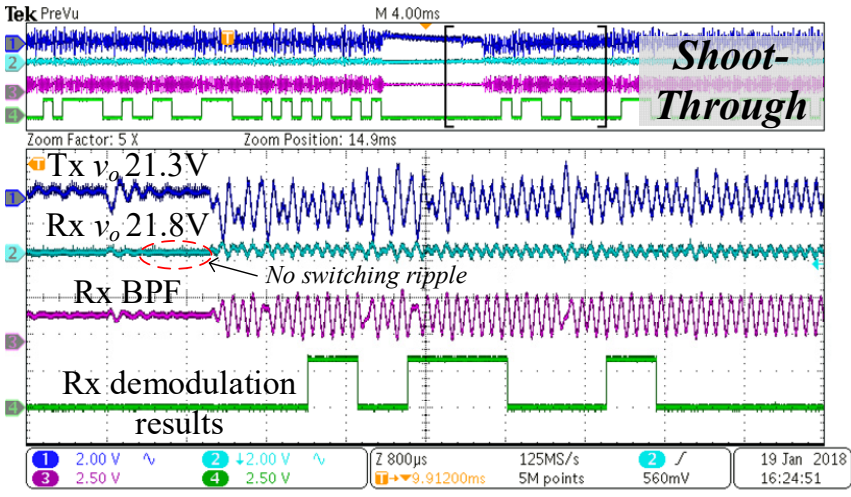

(a)

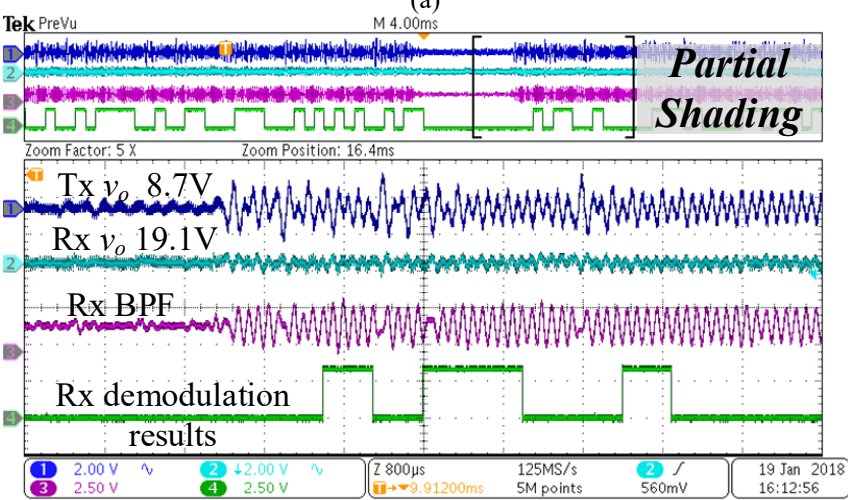

(b)

Fig. 13. (a) Waveform of $\hat{v}_{o}$ at $\mathrm{Tx}$ and $\mathrm{Rx}$, waveform at BPF, and data demodulation results when RXs are working at shoot-through and bus voltage changes into $131 \mathrm{~V}$. (b) Waveform at $\hat{v}_{o}$ of Tx and Rx, waveform at BPF, and data demodulation results when Tx's input current changes into 1.60A.

DCPOs in the string will increase their duty-cycle to balance the dc bus voltage. In this experiment, the input current of Tx is changed to $1.60 \mathrm{~A}$, and the duty-cycle is changed to 0.4 . Fig. 13(b) shows experimental results of $\hat{v}_{o}$ at $\mathrm{Tx}$ and $\mathrm{Rx}$, and demodulation results at $\mathrm{Rx}$. The waveform shows almost the same as before, and the sequence is demodulated correctly.

Therefore, in either shoot-through or partial shading situation, the amplitude of signal is large enough for demodulation. It shows that the communication system will be reliable in different working conditions.

MPPT is the most important feature of DCPO, thus communication should not interfere with the MPPT process. For Tx, MPPT process and data transmission can be separated by the program of controller, so that they do not affect each other. For $\mathrm{Rx}$, the perturbation caused by communication at DCPO's input is small and can be filtered after sampling, hence it will not impact MPPT process. The detailed waveform of MPPT process is shown in Fig. 14, in which the output power is well regulated by conventional $\mathrm{P} \& \mathrm{O}$ method, while communication is linked at the same time.

The above scheme is cost-efficient. For hardware cost, only a dual-channel amplifier TI-LF353DR is used for voltage sampling and BPF, and the price is 0.14USD. For MCU program, the method needs an ADC interrupt routine operating in every 10us, in which the DFT and decode algorithm is processed. The real processing time of the algorithm for STM32F407 is less

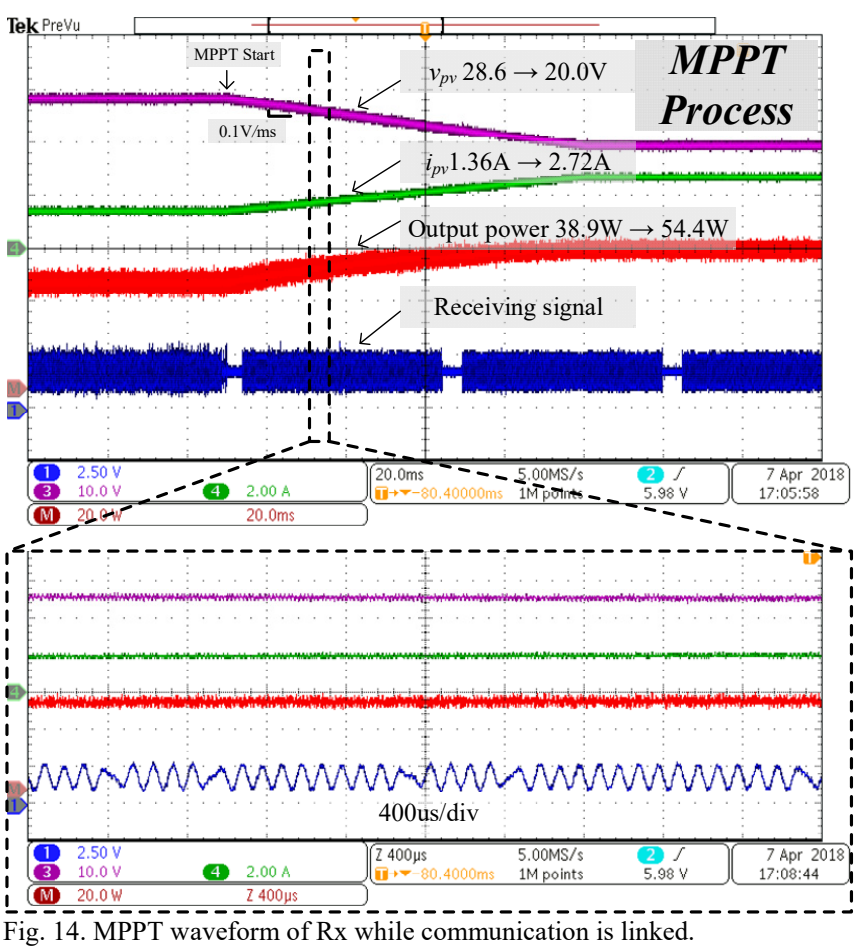

TABLE III

COMPARISON AMONG THE PROPOSED METHOD AND RELATED WORKS

\begin{tabular}{lccc}
\hline \hline Authors [Reference] & $\begin{array}{c}\text { Modem } \\
\text { Scheme }\end{array}$ & $\begin{array}{c}\text { Bit Rate } \\
(\mathrm{kbps})\end{array}$ & $\begin{array}{c}\text { Communication } \\
\text { Cost (USD) }\end{array}$ \\
\hline The proposed method & 2DPSK & 2.0 & 0.14 \\
W. Mao et al [21] & 2ASK & 10.0 & 5.00 \\
E. Roman et al [9] & 2FSK & 2.4 & 12.20 \\
H. Nosato et al [16] & Baseband & 0.005 & 2.0 \\
J. Han et al [20] & 2ASK & 0.7 & Not mentioned \\
\hline \hline
\end{tabular}

than lus. Such demands can be easily satisfied by other commercial MCU chips; thus, the software cost of this method is acceptable in applications. Comparisons among the proposed PLC method and some related conventional PLC methods are presented in TABLE III. It shows that the proposed one has great economic competitiveness in PV industry.

\section{CONCLUSIONS}

This paper presents a novel DCPO design, in which DCPO can not only maximize the PV panel output power in module-level, but also construct a data link among modules. The proposed DCPO design integrates data communication with power conversion. The data is modulated in the power control loop of DCPOs and transmitted to other DCPOs. The technique is easy for implementation, and only a few additional components are required for data communication. A suit of experimental tests has been conducted in a 466W experimental system, where $2 \mathrm{kbps}$ communication among six DCPOs has been achieved, hence the effectiveness of the technique is verified. Comparing with conventional PLC technique in PV system, the proposed method has the advantages of low-cost and integration. Besides, by adjusting the signal carrier wave in MCU program, different amplitudes and forms of the signal can be 
modulated, thus the bit rate can be increased by using advanced modulation methods, e.g. M-QAM.

However, the proposed method has some constraints. First, this PLC method can only link DCPOs in the same string. In a $\mathrm{PV}$ array with several strings connected in parallel, communication among different strings may require extra hardware. Second, due to the modulation mode, the carrier wave frequency is limited to lower than half of switching frequency, hence the communication rate is low comparing with some conventional PLC methods.

\section{REFERENCES}

[1] M. Kasper, D. Bortis and J. W. Kolar, "Classification and comparative evaluation of PV panel-integrated DC-DC converter concepts," IEEE Trans. Power Electron., vol. 29, no. 5, pp. 2511-2526, May. 2014.

[2] G. R. Walker and P. C. Sernia, "Cascaded DC-DC converter connection of photovoltaic modules," IEEE Trans. Power Electron., vol. 19, no. 4, pp. 1130-1139, Jul. 2004.

[3] W. Xiao, N. Ozog and W. G. Dunford, "Topology study of photovoltaic interface for maximum power point tracking," IEEE Trans. Ind. Electron. vol. 54, no. 3, pp. 1696-1704, Jun. 2007.

[4] E. Duran, M. Sidrach-de-Cardona, J. Galan, and J. M. Andujar, "Comparative analysis of buck-boost converters used to obtain I-V characteristic curves of photovoltaic modules," in Proc. PESC, 2008, pp. 2036 2042.

[5] N. Femia, G. Lisi, G. Petrone, G. Spagnuolo and M. Vitelli, "Distributed maximum power point tracking of photovoltaic arrays: Novel approach and system analysis," IEEE Trans. Ind. Electron., vol. 55, no. 7, pp. 2610-2621, Jul. 2008.

[6] N. Femia, G. Petrone, G. Spagnuolo and M. Vitelli, "Optimization of perturb and observe maximum power point tracking method," IEEE Trans. Power Electron., vol. 20, no. 4, pp. 963-973, Jul. 2005.

[7] O. Khan and W. Xiao, "An efficient modeling technique to simulate and control submodule-integrated PV system for single-phase grid connection," IEEE Trans. Sustain. Energy., vol. 7, no. 1, pp. 96-107, Jan. 2016.

[8] H. S. H. Chung, K. K. Tse, S. Y. R. Hui, C. M. Mok and M. T. Ho, "A novel maximum power point tracking technique for solar panels using a SEPIC or Cuk converter," IEEE Trans. Power Electron., vol. 18, no. 3, pp. 717-724, May. 2003.

[9] E. Roman, R. Alonso, P. Ibanez, S. Elorduizapatarietxe and D. Goitia, "Intelligent PV module for grid-connected PV systems," IEEE Trans. Ind Electron., vol. 53, no. 4, pp. 1066-1073, Jun. 2006.

[10] B. Andò, S. Baglio, A. Pistorio, G. M. Tina and C. Ventura, "Sentinella: smart monitoring of photovoltaic systems at panel level," IEEE Trans. Instrum. Meas., vol. 64, no. 8, pp. 2188-2199, Aug. 2015.

[11] P. Guerriero, F. Di Napoli, G. Vallone, V. d'Alessandro and S. Daliento, "Monitoring and diagnostics of PV plants by a wireless self-powered sensor for individual panels," IEEE J. Photovolt., vol. 6, no. 1, pp. 286-294, Jan. 2016.

[12] P. Guerriero, F. Di Napoli, V. d'Alessandro and S. Daliento, "A wireless controlled circuit for PV panel disconnection in case of fire," in Proc. IEEE Int. Symp. Power Electron. Elect. Drives, Autom. Motion, 2014, pp 982-986.

[13] S. Moon, S. G. Yoon and J. H. Park, "A new low-cost centralized MPPT controller system for multiply distributed photovoltaic power conditioning modules," IEEE Trans. Smart Grid., vol. 6, no. 6, pp. 2649-2658, Nov. 2015 .

[14] M. E. Andreoni López, F. J. Galdeano Mantiñan and M. G. Molina, "Implementation of wireless remote monitoring and control of solar photovoltaic (PV) system," in Proc. 6th IEEE/PES Transm. Distrib.: Latin Amer. Conf. Expo. (T\&D-LA), 2012, pp. 1-6.

[15] A. Haidine, B. Adebisi, A. Treytl, H. Pille, B. Honary and A. Portnoy, "High-speed narrowband PLC in Smart Grid landscape State-of-the-art," in Proc. 15th Int. Symp. Power Line Commun. Its Appl. (ISPLC), 2011, pp. 468-473.

[16] H. Nosato, Y. Kasai, E. Takahashi and M. Murakawa, "A very low-cost low-frequency PLC system based on DS-CDMA for DC power lines," in Proc. 16th Int. Symp. Power Line Commun. Its Appl. (ISPLC), 2012, pp. 398-403.

[17] F. J. Sánchez-Pacheco, P. J. Sotorrio-Ruiz, J. R. Heredia-Larrubia, F. Pérez-Hidalgo and M. Sidrach-de-Cardona, "Low cost DC lines PLC based photovoltaic plants parameters smart monitoring communications and control module," in Proc. 2nd Int. Conf. Power Eng., Energy Electr. Drives., 2011, pp. 1-6.

[18] P. Jonke, C. Eder, J. Stöckl and M. Schwark, "Development of a module integrated photovoltaic monitoring system," in Proc. 39th Annu. Conf. IEEE Ind. Electron. Soc. (IECON), 2013, pp. 8080-8084.

[19] J. Han, C. s. Choi, W. k. Park, I. Lee and S. h. Kim, "PLC-based photovoltaic system management for smart home energy management system," IEEE Trans. Consum. Electron., vol. 60, no. 2, pp. 184-189, May. 2014.

[20] J. Han, I. Lee and S. H. Kim, "User-friendly monitoring system for residential PV system based on low-cost power line communication," IEEE Trans. Consum. Electron., vol. 61, no. 2, pp. 175-180, May. 2015.

[21] W. Mao, X. Zhang, R. Cao, F. Wang, T. Zhao and L. Xu, "A research on power line communication based on parallel resonant coupling technology in PV module monitoring," IEEE Trans. Ind. Electron., vol. 65, no. 3, pp. 2653-2662, Mar. 2018.

[22] J. Wu, C. Li and X. He, "A novel power line communication technique based on power electronics circuit topology, " in Proc. 25th Annu. IEEE Applied Power Electronics Conf. Expo. (APEC), 2010, pp. 681-685.

[23] A. Gupta and S. Mazumder, "Sequential Co-Transmission of High-Frequency Power and Data Signals," IEEE Trans. Ind. Informat., doi: 10.1109/TII.2018.2794373

[24] W. Stefanutti, S. Saggini, P. Mattavelli and M. Ghioni, "Power line communication in digitally controlled DC-DC converters using switching frequency modulation," IEEE Trans. Ind. Electron., vol. 55, no. 4, pp. 1509-1518, Apr. 2008.

[25] J. Du, J. Wu, R. Wang, Z. Lin and X. He, "DC power-line communication based on power/signal dual modulation in phase shift full-bridge converters," IEEE Trans. Power Electron., vol. 32, no. 1, pp. 693-702, Jan. 2017.

[26] R. Wang, Z. Lin, J. Du, J. Wu and X. He, "Direct sequence spread spectrum-based PWM strategy for harmonic reduction and communication," IEEE Trans. Power Electron., vol. 32, no. 6, pp. 4455-4465, June 2017.

[27] J. Wu, J. Du, Z. Lin, Y. Hu, C. Zhao and X. He, "Power conversion and signal transmission integration method based on dual modulation of DCDC converters," IEEE Trans. Ind. Electron., vol. 62, no. 2, pp. 1291-1300, Feb. 2015.

[28] S. Haykin, "Passband digital transmission," in Communication System, 4th ed., New York, NY, USA: Wiley, 2001, pp. 407-409.

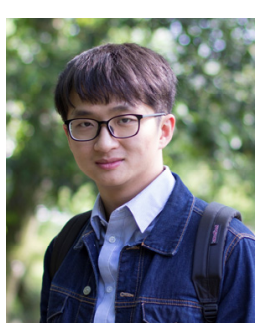

Yue Zhu received the B.S. degree in electrical engineering, Zhejiang University, Hangzhou, China, in 2016, where he is currently a master student in College of Electrical Engineering.

During his study, he focuses on power optimization of renewable generation and communication technique applied in power electronics.

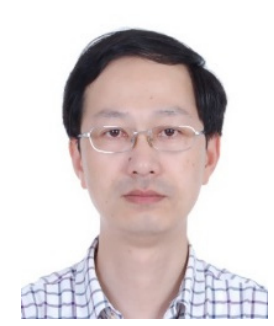

Jiande Wu (M'11) was born in Zhejiang, China, in 1973. He received the B.Sc., M.SC and Ph.D. degree from the College of Electrical Engineering, Zhejiang University, Hangzhou, China, in 1994, 1997 and 2012, respectively. Since 1997, he has been a faculty member at Zhejiang University, where he is currently an associate professor. From 2013 to 2014 , he was an academic visitor at the University of Strathclyde, Glasgow, U.K. His research interests include power electronics control, distributed power electronics system and fieldbus 


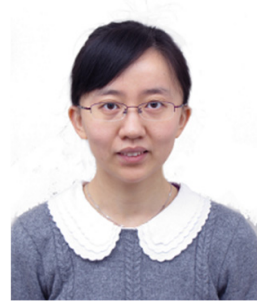

Ruichi Wang (S'14) received the B.S. degree in electrical engineering from Zhejiang University, Hangzhou, China, in 2013. Currently, she is working toward the Ph.D. degree in the College of Electrical Engineering, Zhejiang University, Hangzhou, China She visited Aston University, Birmingham, UK, from September, 2015 to March, 2016. Her current research interests include communication technique applied in power electronics and EMI mitigation for SMPS.

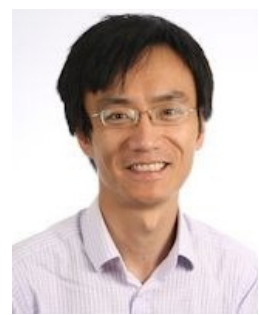

Zhengyu Lin (S'03-M'05-SM'10) received the B.Sc. and M.Sc. degrees from the College of Electrical Engineering, Zhejiang University, Hangzhou, China, in 1998 and 2001, respectively, and the Ph.D. degree from Heriot-Watt University, Edinburgh, U.K.,in 2005. He is currently a Lecturer in Electrical, Electronic and Power Engineering with Aston University, Birmingham, U.K. He was a Research Associate with the University of Sheffield from 2004 to 2006, an R\&D Engineer with Emerson Industrial Automation, Control Techniques PLC from 2006 to 2011, a Senior Research Scientist with Sharp Laboratories of Europe Ltd. from 2011 to 2012, and a Lecturer with Coventry University from 2013 to 2014. His research interests include power electronics and its applications in renewable energy, energy storage, motor drives and power systems.

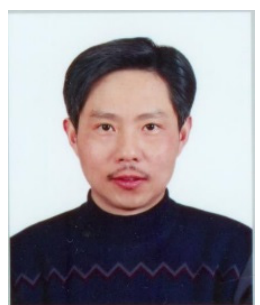

Xiangning He (M'95--SM'96--F'10) received the B.Sc. and M.Sc. degree from Nanjing University of Aeronautical and Astronautical, Nanjing, China, in 1982 and 1985, respectively, and Ph.D. degree from Zhejiang University, Hangzhou, China, in 1989.

In 1991, he obtained a Fellowship from the Royal Society of U.K., and conducted research in Heriot-Watt University, Edinburgh, U.K., as a Post-Doctoral Research Fellow for two years. In 1994, he joined Zhejiang University as an Associate Professor. Since 1996, he has been a Full Professor in the College of Electrical Engineering, Zhejiang University. His research interests are power electronics and their industrial applications.

Dr. He is a Fellow of The Institute of Electrical and Electronics Engineers (IEEE) and has been appointed as IEEE Distinguished Lecturer by the IEEE Power Electronics Society in 2011. He is also a Fellow of the Institution of Engineering and Technology (formerly IEE), U.K. 